\title{
A Modified Three-Term Type CD Conjugate Gradient Algorithm for Unconstrained Optimization Problems
}

\author{
Zhan Wang $\mathbb{D}^{1}{ }^{1}$ Pengyuan Li $\mathbb{D}^{1},{ }^{1}$ Xiangrong Li $\mathbb{D},{ }^{2}$ and Hongtruong Pham ${ }^{3}$ \\ ${ }^{1}$ College of Mathematics and Information Science, Guangxi University, Nanning, Guangxi, China \\ ${ }^{2}$ Business School, College of Mathematics and Information Science, Guangxi University, Nanning, Guangxi, China \\ ${ }^{3}$ Thai Nguyen University of Economics and Business Administration, Thai Nguyen, Vietnam
}

Correspondence should be addressed to Xiangrong Li; xrli68@163.com

Received 26 July 2020; Accepted 13 August 2020; Published 4 September 2020

Guest Editor: Maojun Zhang

Copyright ( $\odot 2020$ Zhan Wang et al. This is an open access article distributed under the Creative Commons Attribution License, which permits unrestricted use, distribution, and reproduction in any medium, provided the original work is properly cited.

Conjugate gradient methods are well-known methods which are widely applied in many practical fields. CD conjugate gradient method is one of the classical types. In this paper, a modified three-term type CD conjugate gradient algorithm is proposed. Some good features are presented as follows: (i) A modified three-term type CD conjugate gradient formula is presented. (ii) The given algorithm possesses sufficient descent property and trust region property. (iii) The algorithm has global convergence with the modified weak Wolfe-Powell (MWWP) line search technique and projection technique for general function. The new algorithm has made great progress in numerical experiments. It shows that the modified three-term type CD conjugate gradient method is more competitive than the classical CD conjugate gradient method.

\section{Introduction}

Considering the problem

$$
\min \left\{f(x) \mid x \in \Re^{n}\right\},
$$

where $f: \Re^{n} \longrightarrow \Re$ is a continuously differentiable function. This kind of model is often used to solve some problems in applied mathematics, economics, engineering, and so on. Generally, the following iteration formula is used to generate the next iteration point:

$$
x_{k+1}=x_{k}+\alpha_{k} d_{k}, \quad k=0,1,2, \ldots,
$$

where $x_{k+1}$ and $x_{k}$ denote the next iteration point and current iteration point, respectively. $\alpha_{k}$ is a step-length and $d_{k}$ is the search direction. The search direction $d_{k}$ generated by Conjugated Gradient (CG) method is defined by the following formula:

$$
d_{k+1}= \begin{cases}-g_{k+1}, & \text { if } k=0, \\ -g_{k+1}+\beta_{k} d_{k}, & \text { if } k \geq 1,\end{cases}
$$

where $g_{k}=g\left(x_{k}\right)$ is the gradient of $f(x)$ at $x_{k}$ and $\beta_{k}$ is a parameter. Different $\beta_{k}$ will generate different CG methods [1-10]. There are six classical forms of $\beta_{k}$ :

$$
\beta_{k}^{P R P}=\frac{g_{k+1}^{T} y_{k}}{\left\|g_{k}\right\|^{2}},[8,9],
$$

$$
\begin{aligned}
& \beta_{k}^{H S}=\frac{g_{k+1}^{T} y_{k}}{y_{k}^{T} d_{k}},[6], \\
& \beta_{k}^{L S}=\frac{g_{k+1}^{T} y_{k}}{-d_{k}^{T} g_{k}},[7], \\
& \beta_{k}^{F R}=\frac{\left\|g_{k+1}\right\|^{2}}{\left\|g_{k}\right\|^{2}},[5], \\
& \beta_{k}^{C D}=\frac{\left\|g_{k+1}\right\|^{2}}{-d_{k}^{T} g_{k}},[4], \\
& \beta_{k}^{D Y}=\frac{\left\|g_{k+1}\right\|^{2}}{y_{k}^{T} d_{k}},[2],
\end{aligned}
$$

where $y_{k}=g_{k+1}-g_{k}$, and $\|\cdot\|$ is the Euclidean norm. Those formulas can be divided into two categories: One includes 
PRP method, HS method, and LS method, which have good numerical performance; the other includes FR method, CD method, and DY method, which have good theory convergence. About these methods, many scholars had applied them to solve nonlinear monotone equations and normal optimization problems, and some good results were achieved [11-16].

Zhang et al. [17] presented a modified PRP CG formula as follows:

$$
d_{k+1}=-g_{k+1}+\frac{g_{k+1}^{T} y_{k} d_{k}-d_{k}^{T} g_{k+1} y_{k}}{g_{k}^{T} g_{k}}
$$

They proved that the modified PRP method is globally convergent with Armijo-type line search.

Yuan et al. [18] proposed another modified PRP CG formula with

$$
d_{k+1}=-\gamma_{k} g_{k+1}+\beta_{k}^{\mathrm{PRP}} d_{k}-\frac{d_{k}^{T} g_{k+1} y_{k}}{\left\|g_{k}\right\|^{2}}
$$

where $\gamma_{k}=\left(\theta_{k} / \min \left\{\eta_{1} s_{k}^{T} y_{k}, \eta_{2} \theta_{k}\right\}\right), \theta_{k}=\left(s_{k}^{T} y_{k} /\left\|s_{k}\right\|^{2}\right), s_{k}=$ $x_{k+1}-x_{k}, \eta_{1} \in(0,1)$. and $\eta_{2} \in(0,1)$. Yuan et al. [18] obtained the global convergence of the modified PRP method with a modified weak Wolfe-Powell (MWWP) line search technique, which was proposed by Yuan et al. [19]:

$$
\begin{aligned}
& f\left(x_{k}+\alpha_{k} d_{k}\right) \leq f_{k}+\lambda \alpha_{k} g_{k}^{T} d_{k}+\alpha_{k} \min \left\{-\lambda_{1} g_{k}^{T} d_{k}, \lambda \frac{\alpha_{k}}{2}\left\|d_{k}\right\|^{2}\right\}, \\
& g\left(x_{k}+\alpha_{k} d_{k}\right)^{T} d_{k} \geq \mu g_{k}^{T} d_{k}+\min \left\{-\lambda_{1} g_{k}^{T} d_{k}, \lambda \alpha_{k}\left\|d_{k}\right\|^{2}\right\},
\end{aligned}
$$

where $\lambda \in(0,(1 / 2)), \lambda_{1} \in(0, \lambda)$, and $\mu \in(\lambda, 1)$. Yuan et al. [16] obtained the global convergence of PRP method by using the above modified weak Wolfe-Powell line search technique and a projection technique:

$$
x_{k+1}=x_{k}+\frac{g\left(\psi_{k}\right)^{T}\left(\psi_{k}-x_{k}\right)+\chi\left\|\psi_{k}-x_{k}\right\|^{2}}{\left\|g\left(\psi_{k}\right)-g\left(x_{k}\right)\right\|^{2}}\left[g\left(\psi_{k}\right)-g\left(x_{k}\right)\right] \text {, }
$$

where $\psi_{k}=x_{k}+\alpha_{k} d_{k}$ is the next point and parameter $\chi>0$. With this projection technique, any unsatisfactory iteration points generated by the normal PRP algorithm will be projected onto a surface to overcome the failure to converge.

Motivated by the above researches, a modified threeterm CD conjugate gradient algorithm is presented with (7), (8), and (9) in this paper. Some good properties are obtained as follows:

(i) A modified three-term type CD conjugate gradient formula is presented

(ii) The given algorithm possesses sufficient descent property and trust region property

(iii) The algorithm has global convergence with the MWWP line search technique and projection technique for general function
This paper is organized as follows: the next section will introduce the modified CD formula and relative algorithm; section 3 gives the proof of the global convergence of the new algorithm; numerical experiments are given in section 4; some conclusions are presented in section 5. Throughout this paper, we use $\|\cdot\|$ to denote the Euclidean norm, $g\left(x_{k}\right)$ and $g\left(x_{k+1}\right)$ are replaced by $g_{k}$ and $g_{k+1}$, respectively.

\section{Motivation and Algorithm}

The convergence of CD conjugate gradient method has been proved [4]; however, the numerical results of this method are worse than the PRP method and others. Therefore, it is necessary to propose a new search direction to improve the numerical performance of the CD method. Meanwhile, the sufficient descent property is significant for obtaining the convergence of the conjugate gradient method:

$$
g_{k}^{T} d_{k} \leq-\eta\left\|g_{k}\right\|^{2}
$$

Then, we also hope that we can propose a new method that possesses this property. Inspired by the above discussion, a modified three-term type $C D$ conjugate gradient formula is designed by the following:

$$
d_{k+1}= \begin{cases}-g_{k+1}, & \text { if } k=0, \\ -\gamma_{k} g_{k+1}+\frac{g_{k+1}^{T} g_{k+1} d_{k}}{\max \left\{-d_{k}^{T} g_{k}, \eta_{1}\left\|g_{k+1}\right\|\left\|d_{k}\right\|\right\}}-\frac{g_{k+1}^{T} d_{k} g_{k+1}}{\max \left\{-d_{k}^{T} g_{k}, \eta_{1}\left\|g_{k+1}\right\|\left\|d_{k}\right\|\right\}}, & \text { if } k \geq 1,\end{cases}
$$


where $\quad \gamma_{k}=\left(\theta_{k} / \min \left\{\eta_{2} s_{k}^{T} y_{k}, \eta_{3} \theta_{k}\right\}\right), \theta_{k}=\left\|s_{k}\right\|^{2}, s_{k}=x_{k+1}-$ $x_{k}, y_{k}=g_{k+1}-g_{k}, \eta_{1}>0$, and $\eta_{2}, \eta_{3} \in(0,1)$. The steps of the given algorithm are listed as follows.

\section{Convergence Analysis}

In this section, we are going to analyse the convergence of the proposed algorithm. The following assumptions are needed.

\section{Assumption 1}

(i) The level set $\Omega=\left\{x \in \mathfrak{R}^{n}: f(x) \leq f\left(x_{0}\right)\right\}$ is bounded.

(ii) $f(x)$ is twice continuously differentiable and bounded below, and the gradient function $g(x)$ is
Lipschitz continuous, which means that there exists a constant $L>0$ such that

$\|g(x)-g(y)\| \leq L\|x-y\|, \quad$ for all $x, y \in \Omega$.

Lemma 1. Let the search direction $d_{k}$ be generated by formula (11), then, the following relations hold:

$$
g_{k+1}^{T} d_{k+1} \leq-\eta\left\|g_{k+1}\right\|^{2} \text {. }
$$

$$
\left\|d_{k+1}\right\| \leq \xi\left\|g_{k+1}\right\|
$$

where constants $\eta, \xi>0$.

Proof. According to the definition of $d_{k}$,

$$
\begin{aligned}
g_{k+1}^{T} d_{k+1} & =g_{k+1}^{T}\left[-\gamma_{k} g_{k+1}+\frac{g_{k+1}^{T} g_{k+1} d_{k}}{\max \left\{-d_{k}^{T} g_{k}, \eta_{1}\left\|g_{k+1}\right\|\left\|d_{k}\right\|\right\}}-\frac{g_{k+1}^{T} d_{k} g_{k+1}}{\max \left\{-d_{k}^{T} g_{k}, \eta_{1}\left\|g_{k+1}\right\|\left\|d_{k}\right\|\right\}}\right] \\
& =-\gamma_{k}\left\|g_{k+1}\right\|^{2}+\frac{\left\|g_{k+1}\right\|^{2} g_{k+1}^{T} d_{k}}{\max \left\{-d_{k}^{T} g_{k}, \eta_{1}\left\|g_{k+1}\right\|\left\|d_{k}\right\|\right\}}-\frac{g_{k+1}^{T} d_{k}\left\|g_{k+1}\right\|^{2}}{\max \left\{-d_{k}^{T} g_{k}, \eta_{1}\left\|g_{k+1}\right\|\left\|d_{k}\right\|\right\}} \\
& =-\gamma_{k}\left\|g_{k+1}\right\|^{2} .
\end{aligned}
$$

For the parameter $\gamma_{k}$, we have the following:

$$
\gamma_{k}=\frac{\theta_{k}}{\min \left\{\eta_{2} s_{k}^{T} y_{k}, \eta_{3} \theta_{k}\right\}} \geq \frac{\theta_{k}}{\eta_{3} \theta_{k}}=\frac{1}{\eta_{3}},
$$

then $g_{k+1}^{T} d_{k+1} \leq-\left(1 / \eta_{3}\right)\left\|g_{k+1}\right\|^{2}$ holds. Letting $\eta=\left(1 / \eta_{3}\right)$, the relation (13) is obtained.

Using the definition of the parameter $\gamma_{k}$, we analyse the value of $\gamma_{k}$ in two cases:

Case 1. $\min \left\{\eta_{2} s_{k}^{T} y_{k}, \eta_{3} \theta_{k}\right\}=\eta_{2} s_{k}^{T} y_{k}$. Similar to Lemma 1 of [20], $s_{k}^{T} y_{k} \geq \rho\left\|s_{k}\right\|^{2}$, where $\rho>0$ is a scalar; then

$$
\gamma_{k}=\frac{\theta_{k}}{\min \left\{\eta_{2} s_{k}^{T} y_{k}, \eta_{3} \theta_{k}\right\}}=\frac{\theta_{k}}{\eta_{2} s_{k}^{T} y_{k}} \leq \frac{\theta_{k}}{\eta_{2} \rho\left\|s_{k}\right\|^{2}}=\frac{\left\|s_{k}\right\|^{2}}{\eta_{2} \rho\left\|s_{k}\right\|^{2}}=\frac{1}{\eta_{2} \rho} .
$$

Case 2. $\min \left\{\eta_{2} s_{k}^{T} y_{k}, \eta_{3} \theta_{k}\right\}=\eta_{3} \theta_{k}$. Then

$$
\gamma_{k}=\frac{\theta_{k}}{\min \left\{\eta_{2} s_{k}^{T} y_{k}, \eta_{3} \theta_{k}\right\}}=\frac{\theta_{k}}{\eta_{3} \theta_{k}}=\frac{1}{\eta_{3}} .
$$
Thus,

$$
\begin{aligned}
\left\|d_{k+1}\right\| & =\left\|-\gamma_{k} g_{k+1}+\frac{g_{k+1}^{T} g_{k+1} d_{k}}{\max \left\{-d_{k}^{T} g_{k}, \eta_{1}\left\|g_{k+1}\right\|\left\|d_{k}\right\|\right\}}-\frac{g_{k+1}^{T} d_{k} g_{k+1}}{\max \left\{-d_{k}^{T} g_{k}, \eta_{1}\left\|g_{k+1}\right\|\left\|d_{k}\right\|\right\}}\right\| \\
& \leq \gamma_{k}\left\|g_{k+1}\right\|+\frac{\left\|g_{k+1}\right\|\left\|g_{k+1}\right\|\left\|d_{k}\right\|}{\max \left\{-d_{k}^{T} g_{k}, \eta_{1}\left\|g_{k+1}\right\|\left\|d_{k}\right\|\right\}}+\frac{\left\|g_{k+1}\right\|\left\|d_{k}\right\|\left\|g_{k+1}\right\|}{\max \left\{-d_{k}^{T} g_{k}, \eta_{1}\left\|g_{k+1}\right\|\left\|d_{k}\right\|\right\}} \\
& \leq \gamma_{k}\left\|g_{k+1}\right\|+\frac{\left\|g_{k+1}\right\|\left\|g_{k+1}\right\|\left\|d_{k}\right\|}{\eta_{1}\left\|g_{k+1}\right\|\left\|d_{k}\right\|}+\frac{\left\|g_{k+1}\right\|\left\|d_{k}\right\|\left\|g_{k+1}\right\|}{\eta_{1}\left\|g_{k+1}\right\|\left\|d_{k}\right\|} \\
& \leq \gamma_{k}\left\|g_{k+1}\right\|+\frac{2}{\eta_{1}}\left\|g_{k+1}\right\| \\
& \leq\left(\xi_{0}+\frac{2}{\eta_{1}}\right)\left\|g_{k+1}\right\| .
\end{aligned}
$$


Step 1: Given the initial point $x_{0} \in \mathfrak{R}^{n}$, constants $\epsilon \in(0,1), 0<\lambda_{1}<(\lambda / 2)<\lambda<(1 / 2), \lambda<\mu<1, \eta_{1}>0, \eta_{2}, \eta_{3} \in(0,1)$, let $d_{0}=-g_{0}$, $k:=0$.

Step 2: If $\left\|g_{k}\right\| \leq \varepsilon$, then, stop; otherwise, proceed to the next step.

Step 3: Compute step size $\alpha_{k}$ by the line search (7) and (8).

Step 4: Let $\psi_{k}=x_{k}+\alpha_{k} d_{k}$.

Step 5: If $-\lambda_{1} g_{k}^{T} d_{k}>\lambda \alpha_{k}\left\|d_{k}\right\|^{2}$ holds, let $x_{k+1}=\psi_{k}, y_{k}=g_{k+1}-g_{k}$, and go to Step 7; otherwise, go to Step 6 .

Step 6: Let $x_{k+1}$ be defined by (9), $y_{k}^{*}=g\left(\psi_{k}\right)-g\left(x_{k}\right)$, and $y_{k}=y_{k}^{*}$.

Step 7: If $\left\|g_{k+1}\right\| \leq \varepsilon$, stop; otherwise, proceed to next step.

Step 8: Calculate the search direction $d_{k}$ by (11).

Step 9: Let $k:=k+1$, and go to step 2 .

Algorithm 1: The modified three-term type CD conjugate gradient algorithm.

Let $\xi=\xi_{0}+\left(2 / \eta_{1}\right) \xi=\xi_{0}+\left(2 / \eta_{1}\right)$; then (14) holds. The proof is complete.

Remark 1. The relation (14) shows that the optimization algorithm possesses the trust region feature.

The following theorem is obtained for the global convergence of Algorithm 1.

Theorem 1. Assume that $\alpha_{k}, d_{k}$ and $x_{k}$ are generated by Algorithm 1 and Lemma 1 holds. Then,

$$
\lim _{k \longrightarrow+\infty}\left\|g_{k}\right\|=0 .
$$

Proof. From the line search (8), we have the following:

$$
g\left(x_{k}+\alpha_{k} d_{k}\right)^{T} d_{k} \geq \mu g_{k}^{T} d_{k}+\min \left\{-\lambda_{1} g_{k}^{T} d_{k}, \lambda \alpha_{k}\left\|d_{k}\right\|^{2}\right\} \geq \mu g_{k}^{T} d_{k} .
$$

According to (ii) of Assumption 1,

$$
\alpha_{k} L\left\|d_{k}\right\|^{2} \geq\left(g_{k+1}-g_{k}\right)^{T} d_{k} \geq-(1-\mu) g_{k}^{T} d_{k} \text {. }
$$

Then, we have the following:

$$
\alpha_{k} \geq \frac{-(1-\mu)}{L\left\|d_{k}\right\|^{2}} g_{k}^{T} d_{k}
$$

Using Lemma 1,

$$
\alpha_{k} \geq \frac{-(1-\mu)}{L\left\|d_{k}\right\|^{2}} g_{k}^{T} d_{k} \geq \frac{(1-\mu) \eta\left\|g_{k}\right\|^{2}}{L\left\|d_{k}\right\|^{2}} \geq \frac{(1-\mu) \eta}{L \xi^{2}}
$$

From Assumption 1, line search (7) and sufficient descent property (13),

$$
\begin{aligned}
f\left(x_{k}+\alpha_{k} d_{k}\right) & \leq f_{k}+\lambda \alpha_{k} g_{k}^{T} d_{k}+\alpha_{k} \min \left\{-\lambda_{1} g_{k}^{T} d_{k}, \lambda \frac{\alpha_{k}}{2}\left\|d_{k}\right\|^{2}\right\} \\
& \leq f_{k}+(\lambda-\lambda)_{1} \alpha_{k} g_{k}^{T} d_{k} \\
& \leq f_{k}-\eta\left(\lambda-\lambda_{1}\right) \alpha_{k}\left\|g_{k}\right\|^{2} \\
& \leq f_{k}-\frac{\eta^{2}\left(\lambda-\lambda_{1}\right)(1-\mu)}{L \xi^{2}}\left\|g_{k}\right\|^{2} .
\end{aligned}
$$

Summing these inequalities from $k=0$ to $+\infty$,

$$
\sum_{k=0}^{\infty}\left\|g_{k}\right\|^{2}<f_{0}-f_{+\infty} .
$$

From Assumption 1, it is easy to know that $f(x)$ is bounded. Then, $\left\|g_{k}\right\| \longrightarrow 0$. The proof is complete.

\section{Numerical Results}

This section will report the numerical experiments with some classical optimization problems, the nonlinear Muskingum model, and the application in image restoration problems. All the tests are coded in MATLAB R2014a, run on a PC with a $2.50 \mathrm{GHz} \mathrm{CPU}$, and $4.00 \mathrm{~GB}$ of memory running the Windows 10 operating system.

4.1. Normal Unconstrained Optimization Problems. In this subsection, the numerical experiments would be done with some test problems from [20], and all test problems are listed in Table 1. We compare Algorithm 1 with the classical CD conjugated method (called Algorithm 2) and the classical PRP conjugated method (called Algorithm 3). The detailed experimental data are list in Table 2. Figures 1-3 show the performance of these three algorithms related to CPU, NI, and NFG. Some columns of Tables 1 and 2 and Figures 1-3 have the following meanings:

(i) No: the serial number of the problem.

(ii) Dim: the dimension of the variable $x$.

(iii) NI: the iteration numbers.

(iv) NFG: the sum of function value and gradient value.

(v) CPU: the calculation time in seconds.

(vi) Dimension: the dimensions are 3000, 9000, and 15000.

(vii) Initialization: the parameters of the algorithms are chosen to be $\lambda=0.1, \lambda_{1}=0.01, \mu=0.9, \eta_{1}=0.01$, $\eta_{2}=0.1, \eta_{3}=0.1, e_{1}=e_{2}=10^{-5}$, and $\epsilon=10^{-6}$, and the initial search direction $d_{0}=-g_{0}$.

(viii) Stop rules: the following Himmeblau stop rule [21] is used: if $\left|f\left(x_{k}\right)\right|>e_{1}$, let stop $1=\left(\mid f\left(x_{k}\right)-f\right.$ $\left.\left(x_{k+1}\right) \mid / f\left(x_{k}\right)\right)$; otherwise, let stop $1=\mid f\left(x_{k}\right)$ $-f\left(x_{k+1}\right) \mid$. For every problem, if the conditions 
TABLE 1: Test problems.

\begin{tabular}{|c|c|}
\hline No & Test problems \\
\hline 1 & Extended freudenstein and roth function \\
\hline 2 & Extended trigonometric function \\
\hline 3 & Extended rosenbrock function \\
\hline 4 & Extended white and holst function \\
\hline 5 & Extended beale function \\
\hline 6 & Extended penalty function \\
\hline 7 & Perturbed quadratic function \\
\hline 8 & Raydan 1 function \\
\hline 9 & Raydan 2 function \\
\hline 10 & Diagonal 1 function \\
\hline 11 & Diagonal 2 function \\
\hline 12 & Diagonal 3 function \\
\hline 13 & Hager function \\
\hline 14 & Generalized tridiagonal 1 function \\
\hline 15 & Extended tridiagonal 1 function \\
\hline 16 & Extended three exponential terms function \\
\hline 17 & Generalized tridiagonal 2 function \\
\hline 18 & Diagonal 4 function \\
\hline 19 & Diagonal 5 function \\
\hline 20 & Extended himmelblau function \\
\hline 21 & Generalized PSC1 function \\
\hline 22 & Extended PSC1 function \\
\hline 23 & Extended powell function \\
\hline 24 & Extended block diagonal BD1 function \\
\hline 25 & Extended maratos function \\
\hline 26 & Extended cliff function \\
\hline 27 & Quadratic diagonal perturbed function \\
\hline 28 & Extended wood function \\
\hline 29 & Extended hiebert function \\
\hline 30 & Quadratic function QF1 function \\
\hline 31 & Extended quadratic penalty QP1 function \\
\hline 32 & Extended quadratic penalty QP2 function \\
\hline 33 & A quadratic function QF2 function \\
\hline 34 & Extended EP1 function \\
\hline 35 & Extended Tridiagonal-2 function \\
\hline 36 & BDQRTIC function (CUTE) \\
\hline 37 & TRIDIA function (CUTE) \\
\hline 38 & ARWHEAD function (CUTE) \\
\hline 39 & ARWHEAD function (CUTE) \\
\hline 40 & NONDQUAR function (CUTE) \\
\hline 41 & DQDRTIC function (CUTE) \\
\hline 42 & EG2 function (CUTE) \\
\hline 43 & DIXMAANA function (CUTE) \\
\hline 44 & DIXMAANB function (CUTE) \\
\hline 45 & DIXMAANC function (CUTE) \\
\hline 46 & DIXMAANE function (CUTE) \\
\hline 47 & Partial perturbed quadratic function \\
\hline 48 & Broyden tridiagonal function \\
\hline 49 & Almost perturbed quadratic function \\
\hline 50 & Tridiagonal perturbed quadratic function \\
\hline 51 & EDENSCH function (CUTE) \\
\hline 52 & VARDIM function (CUTE) \\
\hline 53 & STAIRCASE S1 function \\
\hline 54 & LIARWHD function (CUTE) \\
\hline 55 & DIAGONAL 6 function \\
\hline 56 & DIXON3DQ function (CUTE) \\
\hline 57 & DIXMAANF function (CUTE) \\
\hline 58 & DIXMAANG function (CUTE) \\
\hline 59 & DIXMAANH function (CUTE) \\
\hline 60 & DIXMAANI function (CUTE) \\
\hline
\end{tabular}

TABle 1: Continued.

\begin{tabular}{lc}
\hline No & Test problems \\
\hline 61 & DIXMAANJ function (CUTE) \\
62 & DIXMAANK function (CUTE) \\
63 & DIXMAANL function (CUTE) \\
64 & DIXMAAND function (CUTE) \\
65 & ENGVAL1 function (CUTE) \\
66 & FLETCHCR function (CUTE) \\
67 & COSINE function (CUTE) \\
68 & Extended DENSCHNB function (CUTE) \\
69 & DENSCHNF function (CUTE) \\
70 & SINQUAD function (CUTE) \\
71 & BIGGSB1 function (CUTE) \\
72 & Partial perturbed quadratic PPQ2 function \\
73 & Scaled quadratic SQ1 function \\
\hline
\end{tabular}

$\left\|g\left(x_{k}\right)\right\|<\varepsilon$ or stop $1<e_{2}$ are satisfied, then the program is stopped. This program is also stopped when the number of iterations is greater than one thousand.

From the detailed experimental data of Table 2, it is obvious to see that most of the problems can be solved quickly. For most of the problems, it takes less CPU time to solve those problems with the proposed algorithm. Meanwhile, progress has also been made in NI and NFG. Generally, the algorithm of the proposed method is promising versus the other algorithms. About the numerical results, the algorithm of Dolan and More [22] will be used to more directly show the performance profiles of these algorithms. In Figure 1, the curve of Algorithm 1 is always above the other algorithms. In Figure 2, the curves have the same trend. Algorithm 1 almost solves about 97\% of the test problems in $\tau=30$, while Algorithm 2 just solves $89.9 \%$ and Algorithm 3 just solves $87 \%$ in $\tau=30$. Figure 3 shows a similar trend as Figure 2. All the above pictures show that the modified CD conjugate gradient algorithm is more robust and effective compared with the normal CD method and PRP method. In summary, Algorithm 1 is more competitive versus others.

4.2. The Muskingum Model. It is generally known that parameter estimation is a significant task in engineering applications. The nonlinear Muskingum model will be discussed as a common example of such an application in this subsection.

The Muskingum Model [23] is defined by the following:

$$
\begin{aligned}
\min f\left(x_{1}, x_{2}, x_{3}\right)= & \sum_{i=1}^{n-1}\left(\left(1-\frac{\Delta t}{6}\right) x_{1}\left(x_{2} I_{i+1}+\left(1-x_{2}\right) Q_{i+1}\right)^{x_{3}}\right. \\
& -\left(1-\frac{\Delta t}{6}\right) x_{1}\left(x_{2} I_{i}+\left(1-x_{2}\right) Q_{i}\right)^{x_{3}} \\
& \left.-\frac{\Delta t}{2}\left(I_{i}-Q_{i}\right)+\frac{\Delta t}{2}\left(1-\frac{\Delta t}{3}\right)\left(I_{i+1}-Q_{i+1}\right)\right)^{2} .
\end{aligned}
$$


TABLe 2: The numerical results of Algorithms 1-3.

\begin{tabular}{|c|c|c|c|c|c|c|c|c|c|c|}
\hline \multirow{2}{*}{ No. } & \multirow{2}{*}{ Dim } & \multicolumn{3}{|c|}{ Algorithm 1} & \multicolumn{3}{|c|}{ Algorithm 2} & \multicolumn{3}{|c|}{ Algorithm 3} \\
\hline & & NI & NFG & CPU time & $\mathrm{NI}$ & NFG & CPU time & $\mathrm{NI}$ & NFG & CPU time \\
\hline 1 & 3000 & 7 & 41 & 0.484375 & 20 & 73 & 0.078125 & 16 & 65 & 0.109375 \\
\hline 1 & 9000 & 13 & 89 & 0.328125 & 20 & 70 & 0.265625 & 16 & 62 & 0.28125 \\
\hline 1 & 15000 & 7 & 41 & 0.203125 & 42 & 144 & 0.8125 & 20 & 85 & 0.5625 \\
\hline 2 & 3000 & 169 & 1138 & 1.46875 & 83 & 260 & 0.328125 & 89 & 361 & 0.484375 \\
\hline 2 & 9000 & 108 & 396 & 1.34375 & 91 & 286 & 0.875 & 89 & 298 & 0.890625 \\
\hline 2 & 15000 & 125 & 590 & 2.765625 & 93 & 294 & 1.328125 & 95 & 306 & 1.453125 \\
\hline 3 & 3000 & 9 & 49 & 0.03125 & 109 & 415 & 0.203125 & 32 & 106 & 0.0625 \\
\hline 3 & 9000 & 5 & 26 & 0.078125 & 91 & 348 & 0.265625 & 6 & 29 & 0.015625 \\
\hline 3 & 15000 & 5 & 26 & 0.015625 & 96 & 371 & 0.4375 & 9 & 50 & 0.078125 \\
\hline 4 & 3000 & 43 & 200 & 0.203125 & 167 & 572 & 0.515625 & 29 & 104 & 0.109375 \\
\hline 4 & 9000 & 21 & 91 & 0.203125 & 51 & 202 & 0.515625 & 29 & 109 & 0.21875 \\
\hline 4 & 15000 & 21 & 91 & 0.359375 & 45 & 176 & 0.625 & 43 & 164 & 0.625 \\
\hline 5 & 3000 & 2 & 14 & 0.015625 & 37 & 130 & 0.109375 & 88 & 367 & 0.6875 \\
\hline 5 & 9000 & 41 & 128 & 0.296875 & 36 & 125 & 0.28125 & 29 & 109 & 0.71875 \\
\hline 5 & 15000 & 3 & 26 & 0.046875 & 38 & 131 & 0.421875 & 53 & 171 & 0.734375 \\
\hline 6 & 3000 & 92 & 327 & 0.171875 & 113 & 358 & 0.15625 & 124 & 391 & 0.3125 \\
\hline 6 & 9000 & 104 & 340 & 0.390625 & 133 & 420 & 0.40625 & 144 & 453 & 0.5625 \\
\hline 6 & 15000 & 116 & 370 & 0.65625 & 151 & 474 & 0.640625 & 145 & 466 & 0.765625 \\
\hline 7 & 3000 & 423 & 4746 & 2.40625 & 968 & 3023 & 1.203125 & 1000 & 3041 & 1.265625 \\
\hline 7 & 9000 & 102 & 373 & 0.359375 & 1000 & 3075 & 2.453125 & 1000 & 3062 & 3.09375 \\
\hline 7 & 15000 & 272 & 987 & 1.578125 & 214 & 718 & 0.921875 & 1000 & 3046 & 4.296875 \\
\hline 8 & 3000 & 18 & 65 & 0.046875 & 23 & 74 & 0.03125 & 25 & 79 & 0.046875 \\
\hline 8 & 9000 & 17 & 62 & 0.078125 & 23 & 74 & 0.09375 & 25 & 79 & 0.109375 \\
\hline 8 & 15000 & 17 & 62 & 0.15625 & 23 & 74 & 0.125 & 25 & 79 & 0.171875 \\
\hline 9 & 3000 & 19 & 65 & 0.03125 & 9 & 28 & 0.015625 & 3 & 25 & 0.03125 \\
\hline 9 & 9000 & 3 & 26 & 0.046875 & 9 & 28 & 0.046875 & 23 & 100 & 0.09375 \\
\hline 9 & 15000 & 3 & 26 & 0.046875 & 9 & 28 & 0.03125 & 24 & 110 & 0.234375 \\
\hline 10 & 3000 & 2 & 14 & 0.015625 & 2 & 14 & 0.015625 & 2 & 14 & 0 \\
\hline 10 & 9000 & 2 & 14 & 0 & 2 & 14 & 0 & 2 & 14 & 0 \\
\hline 10 & 15000 & 2 & 14 & 0.03125 & 2 & 14 & 0.015625 & 2 & 14 & 0.03125 \\
\hline 11 & 3000 & 3 & 19 & 0.03125 & 75 & 263 & 0.140625 & 4 & 21 & 0 \\
\hline 11 & 9000 & 3 & 19 & 0.03125 & 175 & 593 & 0.828125 & 6 & 36 & 0.0625 \\
\hline 11 & 15000 & 3 & 19 & 0.140625 & 22 & 88 & 0.140625 & 7 & 37 & 0.078125 \\
\hline 12 & 3000 & 17 & 57 & 0.03125 & 15 & 46 & 0.0625 & 17 & 55 & 0.046875 \\
\hline 12 & 9000 & 16 & 54 & 0.125 & 16 & 49 & 0.09375 & 17 & 56 & 0.1875 \\
\hline 12 & 15000 & 16 & 54 & 0.125 & 16 & 49 & 0.109375 & 17 & 56 & 0.15625 \\
\hline 13 & 3000 & 2 & 14 & 0 & 23 & 138 & 0.078125 & 2 & 14 & 0.03125 \\
\hline 13 & 9000 & 2 & 14 & 0.03125 & 3 & 18 & 0.046875 & 2 & 14 & 0 \\
\hline 13 & 15000 & 2 & 14 & 0.015625 & 2 & 14 & 0.015625 & 2 & 14 & 0.0625 \\
\hline 14 & 3000 & 18 & 85 & 0.5625 & 8 & 27 & 0.15625 & 37 & 169 & 1.0625 \\
\hline 14 & 9000 & 7 & 39 & 0.796875 & 4 & 14 & 0.21875 & 45 & 274 & 4.890625 \\
\hline 14 & 15000 & 7 & 41 & 1.296875 & 4 & 14 & 0.359375 & 50 & 343 & 9.75 \\
\hline 15 & 3000 & 17 & 59 & 0.484375 & 52 & 177 & 1.046875 & 33 & 175 & 0.796875 \\
\hline 15 & 9000 & 25 & 83 & 1.328125 & 50 & 169 & 2.65625 & 33 & 175 & 2.359375 \\
\hline 15 & 15000 & 29 & 95 & 2.5625 & 41 & 138 & 3.578125 & 33 & 175 & 3.796875 \\
\hline 16 & 3000 & 3 & 16 & 0 & 13 & 40 & 0.03125 & 40 & 151 & 0.109375 \\
\hline 16 & 9000 & 8 & 23 & 0.046875 & 17 & 52 & 0.0625 & 24 & 73 & 0.09375 \\
\hline 16 & 15000 & 17 & 59 & 0.15625 & 15 & 46 & 0.09375 & 27 & 82 & 0.171875 \\
\hline 17 & 3000 & 2 & 14 & 0.015625 & 40 & 122 & 0.1875 & 53 & 164 & 0.234375 \\
\hline 17 & 9000 & 36 & 148 & 0.578125 & 35 & 107 & 0.375 & 47 & 149 & 0.546875 \\
\hline 17 & 15000 & 5 & 42 & 0.15625 & 27 & 83 & 0.546875 & 49 & 155 & 1.078125 \\
\hline 18 & 3000 & 36 & 163 & 0.078125 & 3 & 12 & 0 & 384 & 4591 & 1.875 \\
\hline 18 & 9000 & 40 & 229 & 0.15625 & 3 & 12 & 0.046875 & 391 & 4675 & 3.65625 \\
\hline 18 & 15000 & 51 & 325 & 0.359375 & 3 & 12 & 0 & 394 & 4711 & 5.421875 \\
\hline 19 & 3000 & 4 & 30 & 0.015625 & 3 & 11 & 0.015625 & 2 & 14 & 0 \\
\hline 19 & 9000 & 4 & 39 & 0.078125 & 3 & 11 & 0 & 2 & 14 & 0.015625 \\
\hline 19 & 15000 & 4 & 39 & 0.09375 & 3 & 11 & 0.046875 & 2 & 14 & 0.015625 \\
\hline 20 & 3000 & 38 & 134 & 0.09375 & 22 & 70 & 0.03125 & 3 & 18 & 0 \\
\hline 20 & 9000 & 40 & 142 & 0.15625 & 30 & 94 & 0.0625 & 3 & 18 & 0.015625 \\
\hline
\end{tabular}


TABle 2: Continued.

\begin{tabular}{|c|c|c|c|c|c|c|c|c|c|c|}
\hline \multirow{2}{*}{ No. } & \multirow{2}{*}{ Dim } & \multicolumn{3}{|c|}{ Algorithm 1} & \multicolumn{3}{|c|}{ Algorithm 2} & \multicolumn{3}{|c|}{ Algorithm 3} \\
\hline & & NI & NFG & CPU time & NI & NFG & CPU time & NI & NFG & CPU time \\
\hline 20 & 15000 & 40 & 137 & 0.1875 & 38 & 118 & 0.125 & 3 & 18 & 0.046875 \\
\hline 21 & 3000 & 21 & 64 & 0.046875 & 38 & 121 & 0.09375 & 27 & 103 & 0.125 \\
\hline 21 & 9000 & 21 & 64 & 0.265625 & 38 & 121 & 0.21875 & 27 & 103 & 0.1875 \\
\hline 21 & 15000 & 21 & 64 & 0.1875 & 38 & 121 & 0.296875 & 26 & 92 & 0.234375 \\
\hline 22 & 3000 & 2 & 14 & 0.015625 & 6 & 35 & 0.0625 & 6 & 28 & 0.09375 \\
\hline 22 & 9000 & 2 & 14 & 0.046875 & 6 & 35 & 0.15625 & 6 & 28 & 0.171875 \\
\hline 22 & 15000 & 2 & 14 & 0.09375 & 6 & 35 & 0.328125 & 6 & 28 & 0.515625 \\
\hline 23 & 3000 & 23 & 109 & 0.078125 & 180 & 610 & 0.546875 & 20 & 71 & 0.09375 \\
\hline 23 & 9000 & 23 & 109 & 0.28125 & 190 & 657 & 1.5 & 1000 & 3003 & 7.625 \\
\hline 23 & 15000 & 23 & 109 & 0.359375 & 206 & 707 & 2.734375 & 1000 & 3003 & 11.65625 \\
\hline 24 & 3000 & 9 & 44 & 0.015625 & 50 & 191 & 0.109375 & 6 & 68 & 0.015625 \\
\hline 24 & 9000 & 9 & 44 & 0.125 & 25 & 107 & 0.125 & 6 & 69 & 0.125 \\
\hline 24 & 15000 & 9 & 44 & 0.125 & 31 & 129 & 0.25 & 6 & 69 & 0.15625 \\
\hline 25 & 3000 & 6 & 27 & 0 & 18 & 53 & 0.046875 & 33 & 98 & 0.046875 \\
\hline 25 & 9000 & 9 & 35 & 0.078125 & 28 & 83 & 0.046875 & 51 & 165 & 0.171875 \\
\hline 25 & 15000 & 6 & 26 & 0.0625 & 34 & 101 & 0.09375 & 15 & 69 & 0.078125 \\
\hline 26 & 3000 & 86 & 273 & 0.28125 & 96 & 305 & 0.171875 & 95 & 304 & 0.15625 \\
\hline 26 & 9000 & 103 & 351 & 0.59375 & 107 & 338 & 0.453125 & 94 & 301 & 0.40625 \\
\hline 26 & 15000 & 96 & 402 & 1.03125 & 98 & 313 & 0.671875 & 95 & 304 & 0.640625 \\
\hline 27 & 3000 & 244 & 2809 & 1.625 & 22 & 94 & 0.03125 & 29 & 119 & 0.046875 \\
\hline 27 & 9000 & 195 & 2290 & 2.40625 & 64 & 256 & 0.203125 & 1000 & 3004 & 3.25 \\
\hline 27 & 15000 & 946 & 3784 & 5.984375 & 50 & 211 & 0.296875 & 21 & 80 & 0.078125 \\
\hline 28 & 3000 & 9 & 46 & 0.03125 & 44 & 151 & 0.09375 & 36 & 126 & 0.078125 \\
\hline 28 & 9000 & 9 & 46 & 0.0625 & 50 & 178 & 0.15625 & 36 & 123 & 0.09375 \\
\hline 28 & 15000 & 9 & 46 & 0.078125 & 37 & 124 & 0.171875 & 34 & 125 & 0.203125 \\
\hline 29 & 3000 & 3 & 18 & 0 & 4 & 25 & 0 & 4 & 24 & 0.046875 \\
\hline 29 & 9000 & 3 & 18 & 0 & 4 & 24 & 0 & 4 & 24 & 0.046875 \\
\hline 29 & 15000 & 3 & 18 & 0.03125 & 4 & 32 & 0.03125 & 4 & 24 & 0.03125 \\
\hline 30 & 3000 & 135 & 1388 & 0.609375 & 1000 & 3063 & 0.921875 & 1000 & 3013 & 1.125 \\
\hline 30 & 9000 & 591 & 4199 & 4.0625 & 1000 & 3066 & 1.9375 & 1000 & 3013 & 2.328125 \\
\hline 30 & 15000 & 107 & 394 & 0.421875 & 1000 & 3083 & 2.609375 & 1000 & 3013 & 3.359375 \\
\hline 31 & 3000 & 32 & 108 & 0.078125 & 40 & 125 & 0.0625 & 44 & 139 & 0.0625 \\
\hline 31 & 9000 & 31 & 98 & 0.09375 & 43 & 136 & 0.109375 & 45 & 142 & 0.125 \\
\hline 31 & 15000 & 33 & 104 & 0.1875 & 44 & 139 & 0.21875 & 48 & 151 & 0.25 \\
\hline 32 & 3000 & 31 & 94 & 0.078125 & 33 & 102 & 0.046875 & 50 & 153 & 0.078125 \\
\hline 32 & 9000 & 27 & 84 & 0.140625 & 64 & 195 & 0.328125 & 41 & 128 & 0.234375 \\
\hline 32 & 15000 & 29 & 90 & 0.265625 & 45 & 140 & 0.3125 & 46 & 143 & 0.34375 \\
\hline 33 & 3000 & 4 & 13 & 0 & 4 & 12 & 0 & 3 & 9 & 0 \\
\hline 33 & 9000 & 2 & 6 & 0.046875 & 2 & 6 & 0 & 2 & 6 & 0 \\
\hline 33 & 15000 & 2 & 6 & 0 & 2 & 6 & 0 & 2 & 6 & 0.015625 \\
\hline 34 & 3000 & 4 & 12 & 0.03125 & 4 & 11 & 0 & 3 & 16 & 0 \\
\hline 34 & 9000 & 4 & 12 & 0.015625 & 5 & 14 & 0.015625 & 7 & 20 & 0.03125 \\
\hline 34 & 15000 & 4 & 12 & 0.015625 & 6 & 17 & 0.03125 & 9 & 26 & 0.09375 \\
\hline 35 & 3000 & 3 & 17 & 0.015625 & 8 & 23 & 0.03125 & 14 & 41 & 0.03125 \\
\hline 35 & 9000 & 3 & 17 & 0.03125 & 12 & 35 & 0.03125 & 21 & 62 & 0.09375 \\
\hline 35 & 15000 & 5 & 14 & 0.03125 & 15 & 44 & 0.078125 & 25 & 74 & 0.140625 \\
\hline 36 & 3000 & 88 & 344 & 1.75 & 20 & 81 & 0.359375 & 7 & 38 & 0.203125 \\
\hline 36 & 9000 & 35 & 149 & 2.125 & 17 & 69 & 0.875 & 7 & 38 & 0.453125 \\
\hline 36 & 15000 & 94 & 394 & 9 & 33 & 123 & 2.796875 & 7 & 38 & 0.84375 \\
\hline 37 & 3000 & 380 & 1413 & 0.734375 & 1000 & 3111 & 1.328125 & 23 & 85 & 0.046875 \\
\hline 37 & 9000 & 321 & 1183 & 1.375 & 344 & 1155 & 1.109375 & 1000 & 3013 & 3.671875 \\
\hline 37 & 15000 & 1000 & 3686 & 6.40625 & 1000 & 3078 & 4.375 & 1000 & 3005 & 4.90625 \\
\hline 38 & 3000 & 94 & 374 & 0.1875 & 9 & 30 & 0.015625 & 5 & 19 & 0.03125 \\
\hline 38 & 9000 & 215 & 858 & 1 & 22 & 74 & 0.046875 & 4 & 14 & 0.03125 \\
\hline 38 & 15000 & 294 & 1173 & 2.125 & 17 & 57 & 0.078125 & 4 & 14 & 0.046875 \\
\hline 39 & 3000 & 6 & 18 & 0 & 18 & 53 & 0.015625 & 38 & 113 & 0.046875 \\
\hline 39 & 9000 & 6 & 18 & 0.03125 & 19 & 56 & 0.03125 & 41 & 122 & 0.109375 \\
\hline 39 & 15000 & 6 & 18 & 0.0625 & 20 & 59 & 0.09375 & 43 & 128 & 0.1875 \\
\hline 40 & 3000 & 59 & 234 & 0.40625 & 1000 & 3116 & 6.046875 & 3 & 19 & 0.046875 \\
\hline
\end{tabular}


TABle 2: Continued.

\begin{tabular}{|c|c|c|c|c|c|c|c|c|c|c|}
\hline \multirow{2}{*}{ No. } & \multirow{2}{*}{ Dim } & \multicolumn{3}{|c|}{ Algorithm 1} & \multicolumn{3}{|c|}{ Algorithm 2} & \multicolumn{3}{|c|}{ Algorithm 3} \\
\hline & & $\mathrm{NI}$ & NFG & CPU time & NI & NFG & CPU time & NI & NFG & CPU time \\
\hline 40 & 9000 & 3 & 18 & 0.0625 & 1000 & 3109 & 16.03125 & 3 & 17 & 0.046875 \\
\hline 40 & 15000 & 3 & 17 & 0.078125 & 1000 & 3102 & 25.765625 & 3 & 17 & 0.078125 \\
\hline 41 & 3000 & 16 & 96 & 0.0625 & 28 & 98 & 0.03125 & 16 & 60 & 0.046875 \\
\hline 41 & 9000 & 23 & 139 & 0.125 & 23 & 78 & 0.0625 & 16 & 60 & 0.0625 \\
\hline 41 & 15000 & 34 & 242 & 0.34375 & 19 & 66 & 0.078125 & 16 & 60 & 0.078125 \\
\hline 42 & 3000 & 6 & 47 & 0.015625 & 4 & 28 & 0 & 6 & 42 & 0.03125 \\
\hline 42 & 9000 & 6 & 47 & 0.078125 & 4 & 28 & 0.046875 & 6 & 42 & 0.03125 \\
\hline 42 & 15000 & 7 & 60 & 0.125 & 4 & 28 & 0.0625 & 6 & 42 & 0.0625 \\
\hline 43 & 3000 & 21 & 188 & 0.296875 & 19 & 60 & 0.21875 & 58 & 186 & 0.421875 \\
\hline 43 & 9000 & 2 & 14 & 0.0625 & 20 & 63 & 0.359375 & 68 & 216 & 1.234375 \\
\hline 43 & 15000 & 24 & 81 & 0.765625 & 20 & 63 & 0.59375 & 72 & 228 & 2.3125 \\
\hline 44 & 3000 & 2 & 14 & 0.015625 & 27 & 84 & 0.203125 & 66 & 216 & 0.4375 \\
\hline 44 & 9000 & 2 & 14 & 0.046875 & 28 & 87 & 0.5 & 75 & 241 & 1.328125 \\
\hline 44 & 15000 & 2 & 14 & 0.078125 & 28 & 87 & 0.78125 & 77 & 247 & 2.3125 \\
\hline 45 & 3000 & 13 & 40 & 0.09375 & 43 & 132 & 0.28125 & 75 & 228 & 0.515625 \\
\hline 45 & 9000 & 13 & 40 & 0.328125 & 45 & 138 & 0.8125 & 79 & 240 & 1.484375 \\
\hline 45 & 15000 & 13 & 40 & 0.375 & 46 & 141 & 1.34375 & 81 & 246 & 2.28125 \\
\hline 46 & 3000 & 86 & 663 & 0.96875 & 60 & 194 & 0.40625 & 24 & 149 & 0.28125 \\
\hline 46 & 9000 & 2 & 14 & 0.046875 & 153 & 510 & 2.90625 & 178 & 1091 & 5.453125 \\
\hline 46 & 15000 & 2 & 14 & 0.078125 & 154 & 519 & 4.703125 & 200 & 1355 & 10.203125 \\
\hline 47 & 3000 & 76 & 230 & 13.859375 & 76 & 229 & 13.515625 & 20 & 71 & 3.71875 \\
\hline 47 & 9000 & 55 & 188 & 92.234375 & 34 & 111 & 53.96875 & 11 & 45 & 17.125 \\
\hline 47 & 15000 & 82 & 304 & 397.6875 & 86 & 276 & 382.3125 & 92 & 306 & 416.421875 \\
\hline 48 & 3000 & 12 & 52 & 0.09375 & 30 & 97 & 0.140625 & 23 & 82 & 0.109375 \\
\hline 48 & 9000 & 119 & 665 & 2.71875 & 35 & 115 & 0.4375 & 37 & 133 & 0.5 \\
\hline 48 & 15000 & 177 & 1485 & 8.859375 & 33 & 109 & 0.6875 & 126 & 383 & 2.546875 \\
\hline 49 & 3000 & 516 & 5422 & 2.796875 & 1000 & 3093 & 0.890625 & 1000 & 3013 & 1.09375 \\
\hline 49 & 9000 & 153 & 560 & 0.5 & 1000 & 3171 & 1.9375 & 1000 & 3013 & 2.390625 \\
\hline 49 & 15000 & 487 & 1800 & 2.109375 & 1000 & 3072 & 2.890625 & 1000 & 3013 & 3.453125 \\
\hline 50 & 3000 & 194 & 1609 & 8.40625 & 1000 & 3025 & 14.765625 & 1000 & 3013 & 15.203125 \\
\hline 50 & 9000 & 320 & 3100 & 43 & 169 & 560 & 8.484375 & 1000 & 3013 & 42.484375 \\
\hline 50 & 15000 & 241 & 888 & 20.484375 & 1000 & 3107 & 71.375 & 1000 & 3013 & 70.25 \\
\hline 51 & 3000 & 49 & 218 & 0.28125 & 16 & 49 & 0.078125 & 28 & 85 & 0.125 \\
\hline 51 & 9000 & 2 & 14 & 0.03125 & 15 & 46 & 0.171875 & 28 & 85 & 0.28125 \\
\hline 51 & 15000 & 2 & 14 & 0.078125 & 15 & 46 & 0.234375 & 28 & 85 & 0.46875 \\
\hline 52 & 3000 & 149 & 474 & 0.421875 & 173 & 548 & 0.46875 & 182 & 575 & 0.453125 \\
\hline 52 & 9000 & 170 & 541 & 1.140625 & 192 & 607 & 1.15625 & 206 & 649 & 1.28125 \\
\hline 52 & 15000 & 178 & 565 & 2.046875 & 201 & 636 & 2.03125 & 215 & 678 & 1.984375 \\
\hline 53 & 3000 & 3 & 26 & 0.03125 & 441 & 1487 & 0.453125 & 3 & 26 & 0.046875 \\
\hline 53 & 9000 & 3 & 27 & 0.015625 & 430 & 1444 & 1.03125 & 56 & 193 & 0.140625 \\
\hline 53 & 15000 & 2 & 14 & 0.03125 & 1000 & 3078 & 3.46875 & 2 & 14 & 0.015625 \\
\hline 54 & 3000 & 381 & 1403 & 0.765625 & 21 & 93 & 0.03125 & 3 & 18 & 0.046875 \\
\hline 54 & 9000 & 48 & 185 & 0.203125 & 98 & 354 & 0.3125 & 3 & 18 & 0.03125 \\
\hline 54 & 15000 & 1000 & 3978 & 7 & 65 & 238 & 0.390625 & 3 & 18 & 0.015625 \\
\hline 55 & 3000 & 19 & 65 & 0.109375 & 14 & 43 & 0.046875 & 3 & 25 & 0.0625 \\
\hline 55 & 9000 & 3 & 26 & 0.09375 & 15 & 46 & 0.171875 & 23 & 100 & 0.390625 \\
\hline 55 & 15000 & 3 & 26 & 0.140625 & 15 & 46 & 0.34375 & 23 & 104 & 0.75 \\
\hline 56 & 3000 & 3 & 16 & 0 & 1000 & 3125 & 0.984375 & 1000 & 2999 & 1.0625 \\
\hline 56 & 9000 & 3 & 16 & 0.046875 & 1000 & 3151 & 2.078125 & 1000 & 2999 & 2.1875 \\
\hline 56 & 15000 & 3 & 16 & 0.015625 & 1000 & 3125 & 3.359375 & 1000 & 2999 & 3.71875 \\
\hline 57 & 3000 & 2 & 14 & 0.015625 & 71 & 226 & 0.5 & 137 & 1398 & 2.203125 \\
\hline 57 & 9000 & 2 & 14 & 0.046875 & 67 & 211 & 1.234375 & 102 & 602 & 3.046875 \\
\hline 57 & 15000 & 2 & 14 & 0.078125 & 69 & 221 & 1.984375 & 210 & 1546 & 10.84375 \\
\hline 58 & 3000 & 13 & 40 & 0.09375 & 109 & 358 & 0.796875 & 584 & 1985 & 3.921875 \\
\hline 58 & 9000 & 13 & 40 & 0.21875 & 119 & 398 & 2.234375 & 22 & 88 & 0.453125 \\
\hline 58 & 15000 & 13 & 40 & 0.390625 & 149 & 490 & 4.65625 & 22 & 88 & 0.6875 \\
\hline 59 & 3000 & 3 & 18 & 0.015625 & 48 & 170 & 0.34375 & 189 & 2038 & 3.140625 \\
\hline 59 & 9000 & 3 & 18 & 0.09375 & 46 & 163 & 0.875 & 239 & 2683 & 10.328125 \\
\hline 59 & 15000 & 3 & 18 & 0.09375 & 54 & 187 & 1.71875 & 256 & 2912 & 17.8125 \\
\hline
\end{tabular}


TABLE 2: Continued.

\begin{tabular}{|c|c|c|c|c|c|c|c|c|c|c|}
\hline \multirow{2}{*}{ No. } & \multirow{2}{*}{ Dim } & \multicolumn{3}{|c|}{ Algorithm 1} & \multicolumn{3}{|c|}{ Algorithm 2} & \multicolumn{3}{|c|}{ Algorithm 3} \\
\hline & & NI & NFG & CPU time & NI & NFG & CPU time & NI & NFG & CPU time \\
\hline 60 & 3000 & 44 & 377 & 1 & 110 & 357 & 0.734375 & 80 & 466 & 0.859375 \\
\hline 60 & 9000 & 2 & 14 & 0.046875 & 184 & 619 & 3.515625 & 97 & 743 & 3.453125 \\
\hline 60 & 15000 & 2 & 14 & 0.078125 & 184 & 624 & 5.859375 & 104 & 1000 & 6.4375 \\
\hline 61 & 3000 & 2 & 14 & 0.015625 & 73 & 236 & 0.515625 & 175 & 1891 & 2.875 \\
\hline 61 & 9000 & 2 & 14 & 0.046875 & 78 & 255 & 1.453125 & 188 & 1193 & 5.796875 \\
\hline 61 & 15000 & 2 & 14 & 0.109375 & 69 & 229 & 2.03125 & 114 & 649 & 4.96875 \\
\hline 62 & 3000 & 3 & 18 & 0.015625 & 86 & 280 & 0.625 & 27 & 103 & 0.1875 \\
\hline 62 & 9000 & 3 & 18 & 0.078125 & 71 & 240 & 1.28125 & 126 & 702 & 3.609375 \\
\hline 62 & 15000 & 3 & 18 & 0.078125 & 47 & 160 & 1.390625 & 75 & 415 & 3.25 \\
\hline 63 & 3000 & 15 & 46 & 0.15625 & 139 & 455 & 1 & 157 & 627 & 1.1875 \\
\hline 63 & 9000 & 15 & 46 & 0.265625 & 252 & 818 & 4.734375 & 72 & 309 & 1.515625 \\
\hline 63 & 15000 & 15 & 46 & 0.453125 & 221 & 742 & 6.953125 & 87 & 363 & 3.046875 \\
\hline 64 & 3000 & 18 & 55 & 0.140625 & 73 & 222 & 0.4375 & 24 & 86 & 0.1875 \\
\hline 64 & 9000 & 18 & 55 & 0.34375 & 77 & 234 & 1.328125 & 24 & 86 & 0.484375 \\
\hline 64 & 15000 & 18 & 55 & 0.578125 & 79 & 240 & 2.328125 & 24 & 86 & 0.859375 \\
\hline 65 & 3000 & 3 & 19 & 0.109375 & 31 & 94 & 0.515625 & 33 & 102 & 0.578125 \\
\hline 65 & 9000 & 3 & 19 & 0.515625 & 30 & 91 & 1.4375 & 31 & 96 & 1.5 \\
\hline 65 & 15000 & 3 & 19 & 0.6875 & 30 & 91 & 2.484375 & 31 & 96 & 2.484375 \\
\hline 66 & 3000 & 6 & 30 & 0.203125 & 4 & 21 & 0.078125 & 4 & 19 & 0.078125 \\
\hline 66 & 9000 & 6 & 30 & 0.5 & 4 & 21 & 0.234375 & 4 & 19 & 0.265625 \\
\hline 66 & 15000 & 120 & 479 & 11.015625 & 4 & 21 & 0.453125 & 4 & 19 & 0.375 \\
\hline 67 & 3000 & 10 & 64 & 0.09375 & 7 & 69 & 0.03125 & 11 & 72 & 0.046875 \\
\hline 67 & 9000 & 7 & 42 & 0.046875 & 38 & 258 & 0.796875 & 11 & 69 & 0.15625 \\
\hline 67 & 15000 & 22 & 136 & 0.890625 & 24 & 170 & 0.71875 & 11 & 67 & 0.234375 \\
\hline 68 & 3000 & 24 & 80 & 0.078125 & 22 & 67 & 0.03125 & 44 & 491 & 0.328125 \\
\hline 68 & 9000 & 2 & 14 & 0 & 23 & 70 & 0.046875 & 2 & 14 & 0.03125 \\
\hline 68 & 15000 & 2 & 14 & 0.0625 & 23 & 70 & 0.078125 & 2 & 14 & 0.03125 \\
\hline 69 & 3000 & 5 & 33 & 0 & 53 & 163 & 0.109375 & 28 & 88 & 0.0625 \\
\hline 69 & 9000 & 5 & 33 & 0.046875 & 51 & 157 & 0.21875 & 20 & 71 & 0.140625 \\
\hline 69 & 15000 & 5 & 33 & 0.046875 & 51 & 157 & 0.359375 & 20 & 71 & 0.171875 \\
\hline 70 & 3000 & 66 & 277 & 1.125 & 43 & 167 & 0.671875 & 13 & 70 & 0.203125 \\
\hline 70 & 9000 & 10 & 45 & 0.5 & 21 & 90 & 1.03125 & 15 & 77 & 0.71875 \\
\hline 70 & 15000 & 68 & 245 & 4.96875 & 9 & 34 & 0.59375 & 25 & 98 & 2.03125 \\
\hline 71 & 3000 & 3 & 16 & 0 & 42 & 133 & 0.046875 & 3 & 16 & 0.046875 \\
\hline 71 & 9000 & 3 & 16 & 0.015625 & 42 & 133 & 0.0625 & 3 & 16 & 0 \\
\hline 71 & 15000 & 3 & 16 & 0.015625 & 42 & 133 & 0.125 & 3 & 16 & 0.046875 \\
\hline 72 & 3000 & 206 & 765 & 41.4375 & 163 & 583 & 31.453125 & 15 & 60 & 2.78125 \\
\hline 72 & 9000 & 1000 & 3694 & 1734.984375 & 1000 & 3036 & 1571.609375 & 15 & 60 & 24.125 \\
\hline 72 & 15000 & 1000 & 3611 & 4739.8125 & 163 & 568 & 751.625 & 15 & 60 & 66.703125 \\
\hline 73 & 3000 & 562 & 6118 & 2.671875 & 320 & 1055 & 0.28125 & 1000 & 3017 & 0.984375 \\
\hline 73 & 9000 & 1000 & 9069 & 8.703125 & 1000 & 3048 & 1.90625 & 1000 & 3017 & 2.375 \\
\hline 73 & 15000 & 798 & 5688 & 6.53125 & 1000 & 3041 & 2.71875 & 1000 & 3017 & 3.078125 \\
\hline
\end{tabular}

Some of the variables have the following meanings:

(i) $n$ : the total time number.

(ii) $x_{1}$ : the storage time constant.

(iii) $I_{i}$ : the observed inflow discharge.

(iv) $x_{2}$ : the weighting factor.

(v) $Q_{i}$ : the observed outflow discharges.

(vi) $x_{3}$ : an additional parameter.

(vii) $\Delta t$ : the time step at time $t_{i}(i=1,2, \ldots, n)$.

In the experiment, the observed data of the flood run-off process from Chenggouwan and Linqing of Nanyunhe River in the Haihe Basin, Tianjin, China, are used. We choose the initial point $x=[0,1,1]^{T}$; detailed data about $I_{i}$ and $Q_{i}$ for the years 1960, 1961, and 1964 were obtained (see [24] in detail). $\Delta t=12(h)$ was selected. The results of these three algorithms are listed in Table 3. The performance of the presented algorithm is shown in Figures 4-6.

Some conclusions are obtained from this experiment: (1) from Figures 4-6, we conclude that we can calculate approximations of flood outflows by using Algorithm 1, and Algorithm 1 is effective for the nonlinear Muskingum model; (2) the final points $\left(x_{1}, x_{2}\right.$ and $\left.x_{3}\right)$ of these three algorithms are interesting, and they are competitive with the final points of similar algorithms; and (3) the final points of Algorithm 1 are different from those of the BFGS method and HIWO method, which implies that the nonlinear Muskingum model has different optimum approximation solutions. 


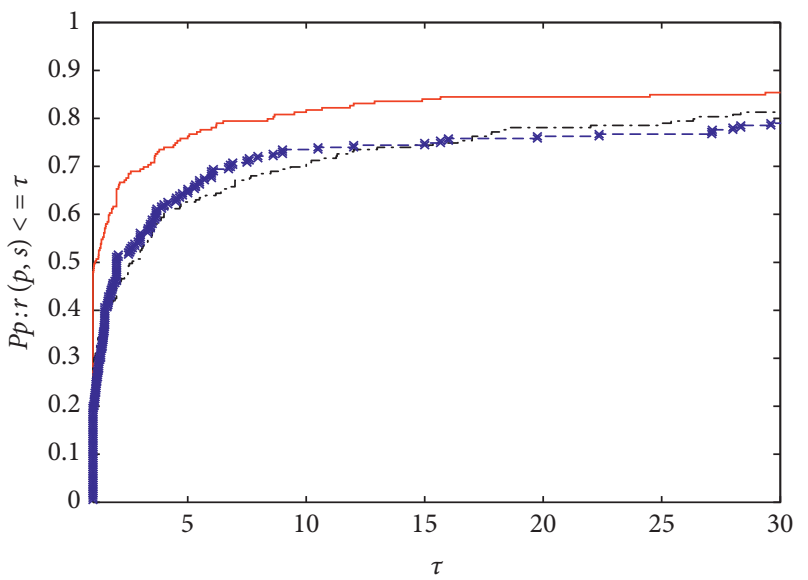

_- Algorithm 1
-.- Algorithm 2
-*- Algorithm 3

FIgURE 1: Performance profiles of these methods (CPU).

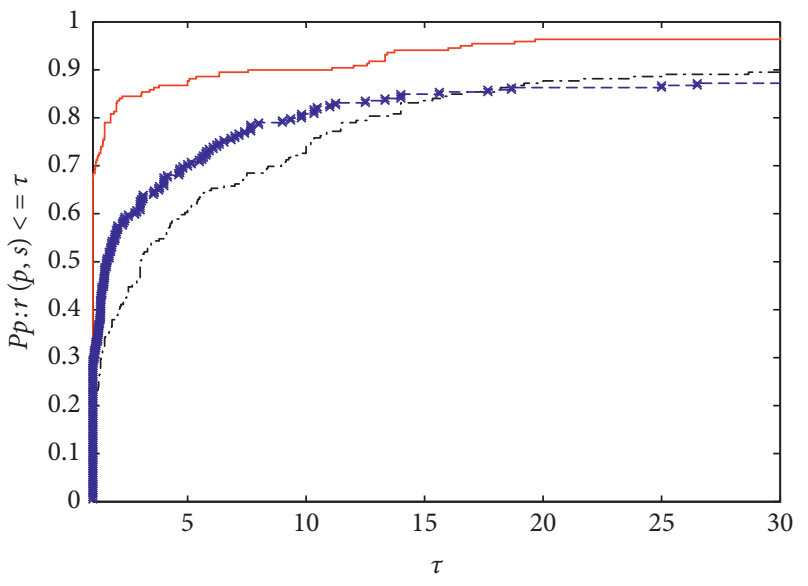

— Algorithm 1

-..- Algorithm 2

-*- Algorithm 3

Figure 2: Performance profiles of these methods (NI).

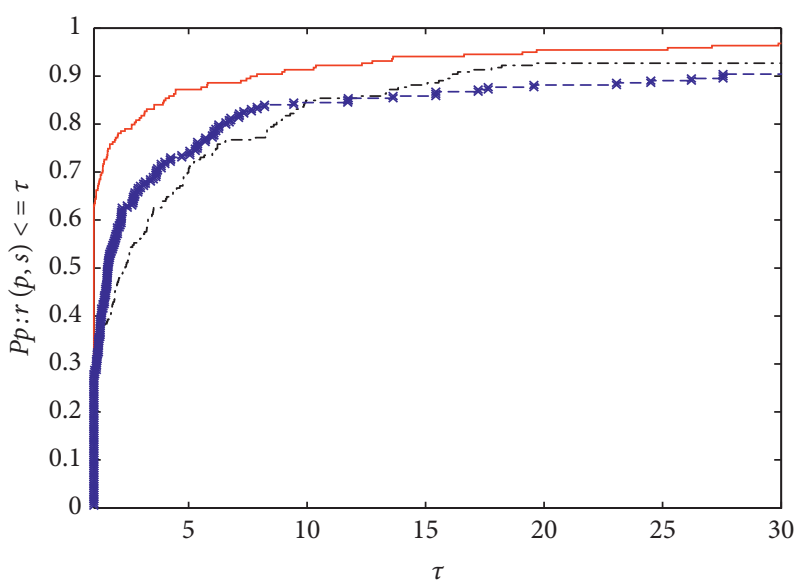

— Algorithm 1

...- Algorithm 2

-*- Algorithm 3

FIgUre 3: Performance profiles of these methods (NGF). 
TABLE 3: Results of the three algorithms.

\begin{tabular}{lccr}
\hline Algorithms & $x_{1}$ & $x_{2}$ & $x_{3}$ \\
\hline BFGS [25] & 10.8156 & 0.9826 & 1.0219 \\
HIWO [23] & 13.2813 & 0.8001 & 0.9933 \\
Algorithm 1 & 11.1848 & 1.0000 & 0.9995 \\
\hline
\end{tabular}

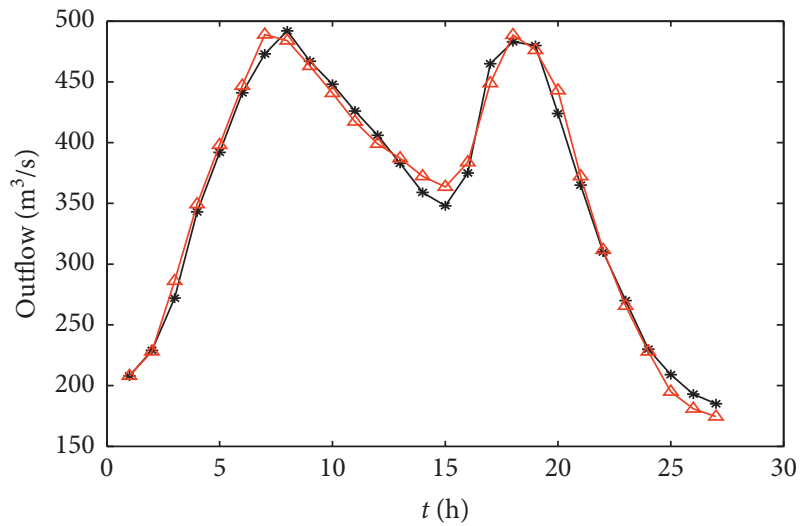

* Observed

$\triangle$ Computed

Figure 4: Performance of date in 1960.

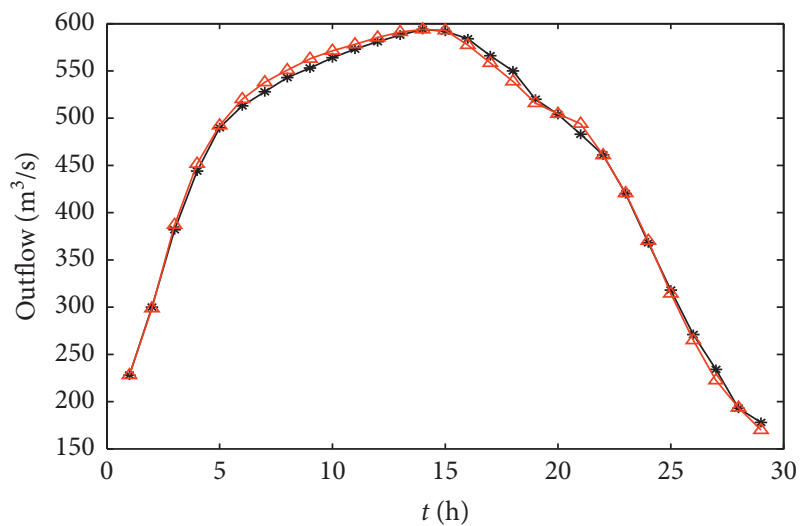

* Observed

$\triangle$ Computed

FIgure 5: Performance of date in 1961.

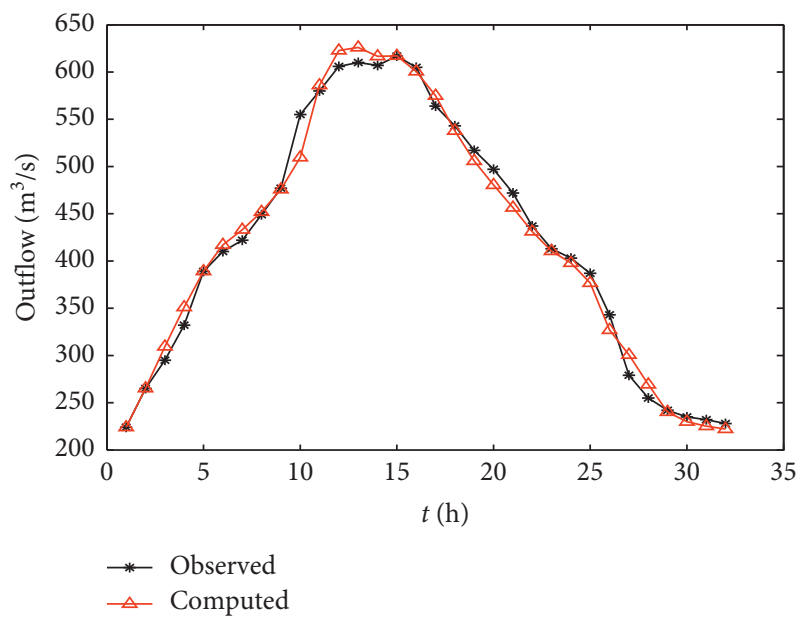

Figure 6: Performance of date in 1961. 

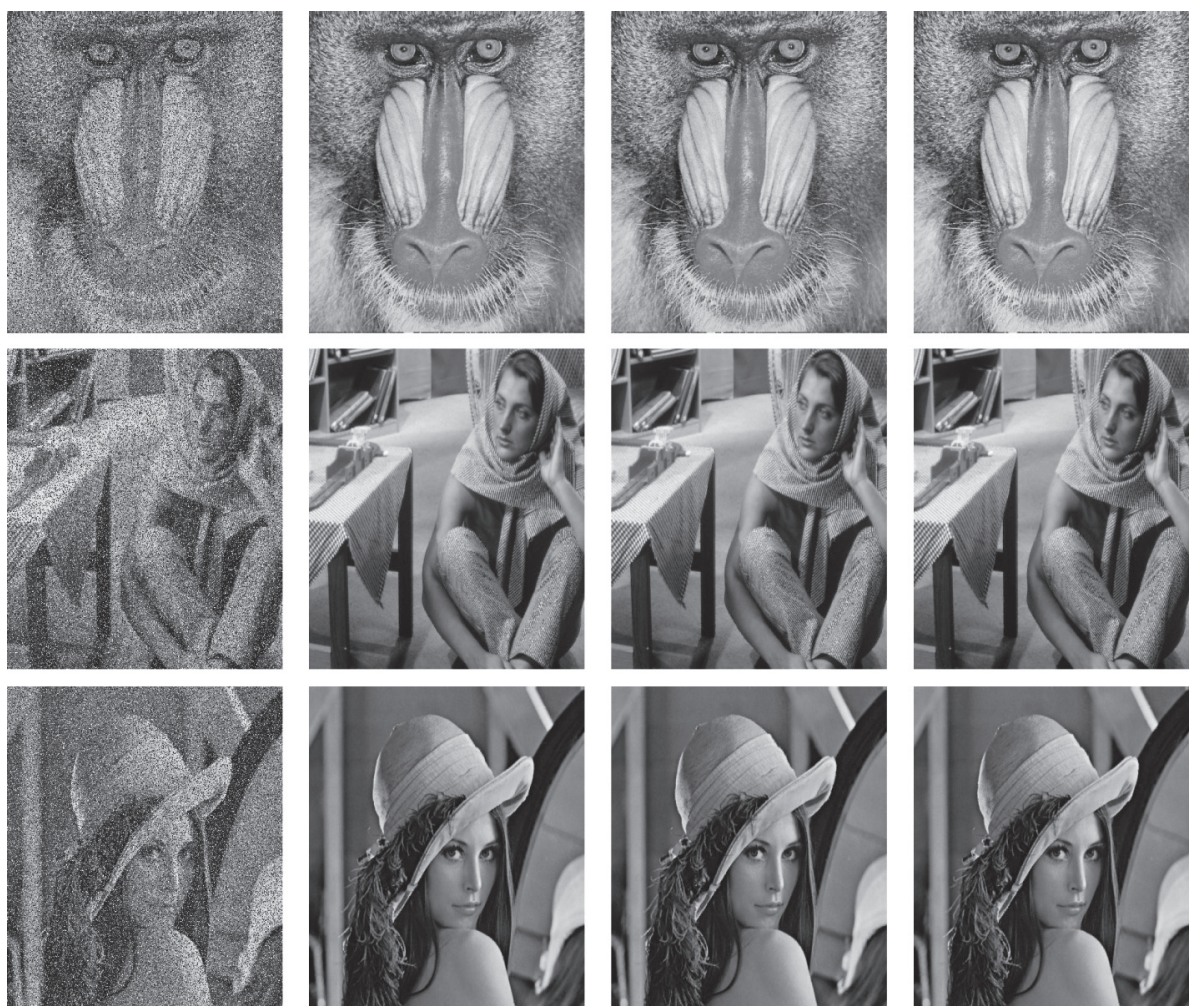

FIgURE 7: Restoration of the Banoon image, Barbara image, and Lena image by Algorithms 1-3. From left to right: a noisy image with 30\% salt-and-pepper noise, the restorations obtained with Algorithms 1-3.
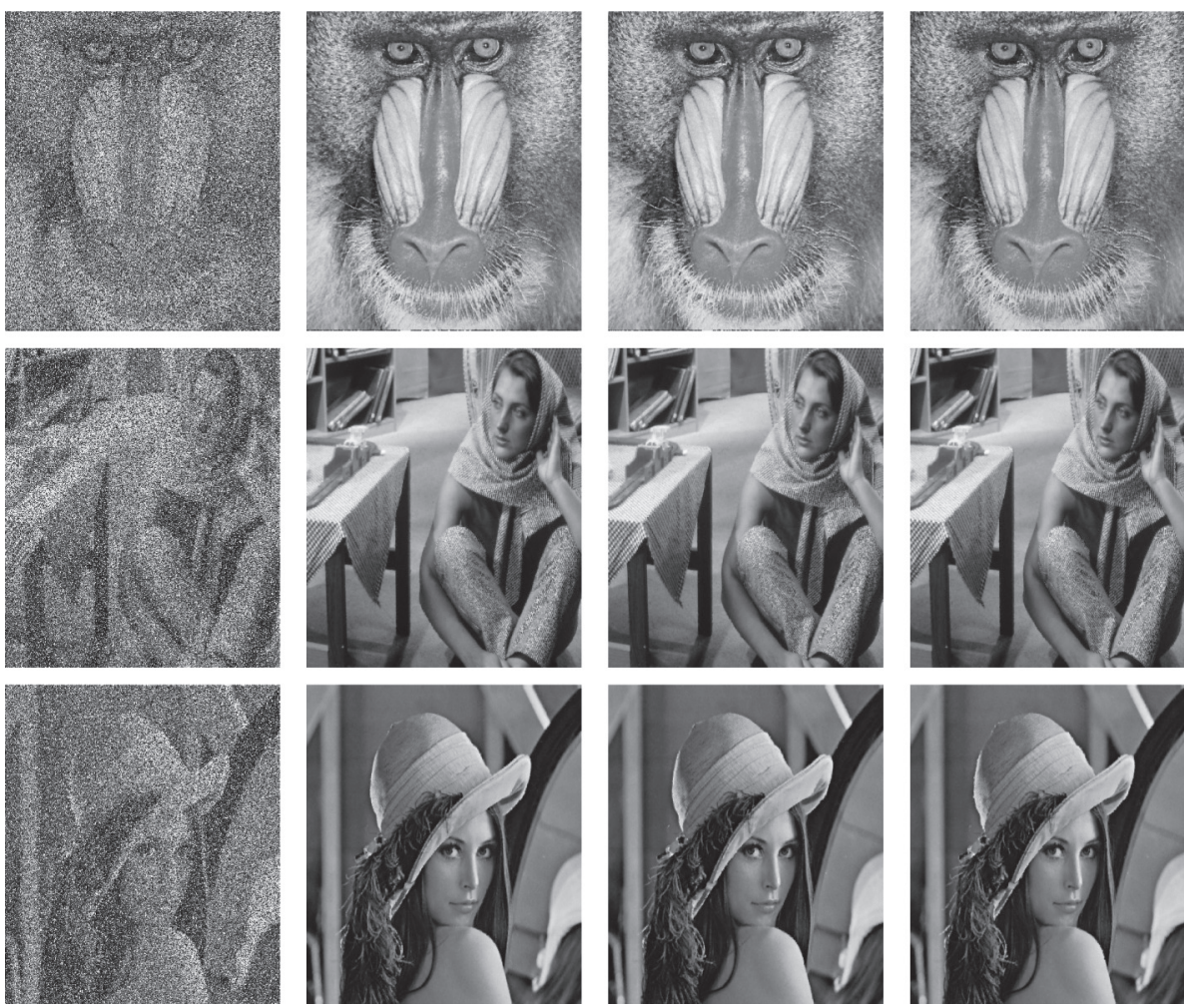

Figure 8: Restoration of the Banoon image, Barbara image, and Lena image by Algorithms 1-3. From left to right: a noisy image with 50\% salt-and-pepper noise, the restorations obtained with the Algorithms 1-3. 

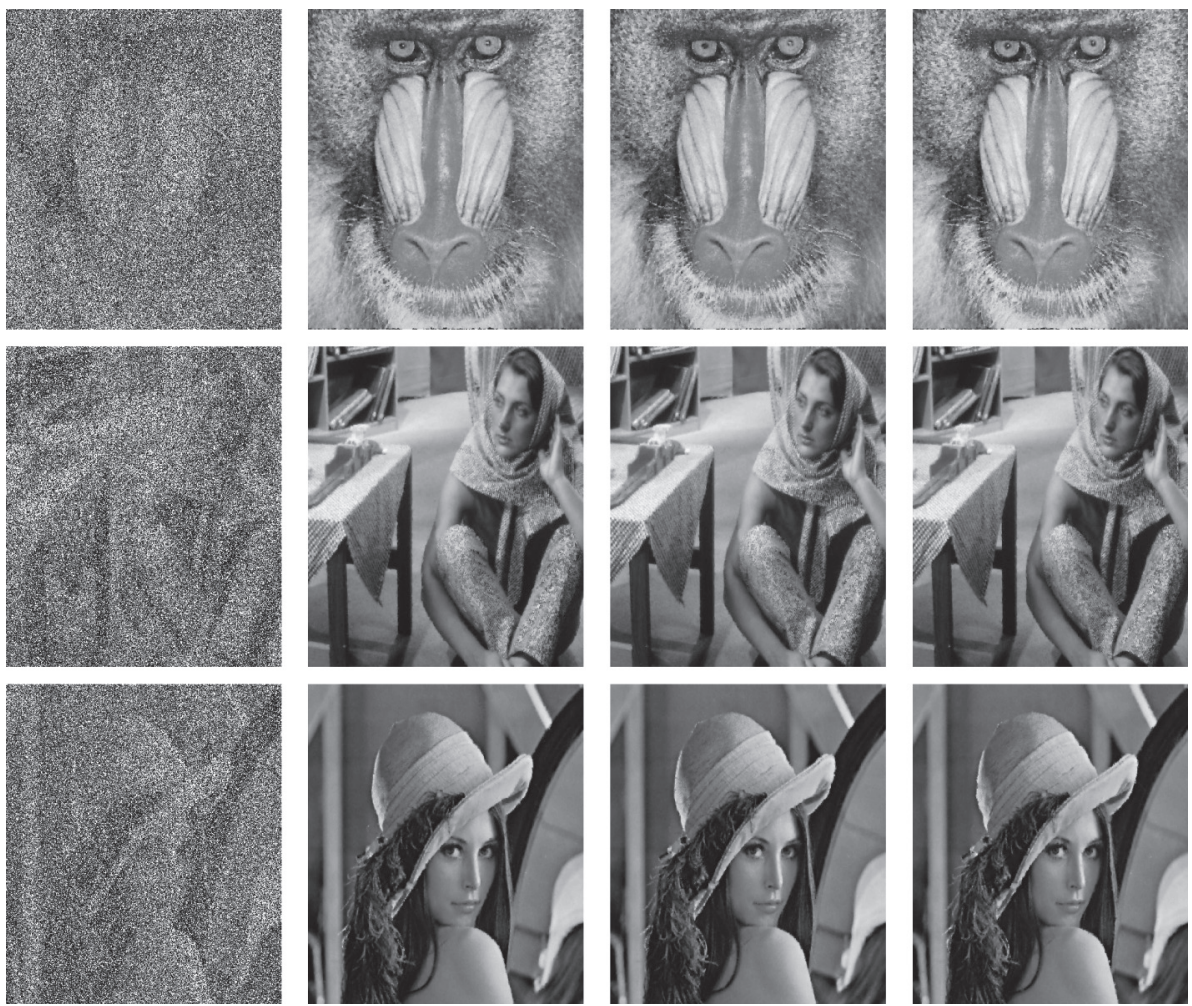

Figure 9: Restoration of the Banoon image, Barbara image, and Lena image by Algorithms 1-3. From left to right: a noisy image with 70\% salt-and-pepper noise, the restorations obtained with the Algorithms 1-3.

TABle 4: CPU time of those algorithms in seconds.

\begin{tabular}{|c|c|c|c|c|}
\hline $30 \%$ noise & Lena & Barbara & Banoon & Total \\
\hline Algorithm 1 & 4.438 & 4.390 & 4.656 & 13.484 \\
\hline Algorithm 2 & 4.563 & 5.234 & 5.406 & 15.203 \\
\hline Algorithm 3 & 5.156 & 6.313 & 6.781 & 18.250 \\
\hline $50 \%$ noise & Lena & Barbara & Banoon & Total \\
\hline Algorithm 1 & 8.609 & 7.891 & 8.141 & 24.641 \\
\hline Algorithm 2 & 9.219 & 10.688 & 10.547 & 30.454 \\
\hline Algorithm 3 & 11.297 & 14.391 & 14.859 & 40.547 \\
\hline $70 \%$ noise & Lena & Barbara & Banoon & Total \\
\hline Algorithm 1 & 15.750 & 10.578 & 11.984 & 38.312 \\
\hline Algorithm 2 & 13.891 & 14.438 & 14.703 & 43.032 \\
\hline Algorithm 3 & 14.859 & 19.922 & 20.375 & 55.156 \\
\hline
\end{tabular}

4.3. Image Restoration Problems. In this subsection, the above algorithms will be applied to image restoration problems. The original images corrupted by impulse noise are treated as objects here. These problems are regarded as one of the most difficult problems in optimization fields. Related parameter settings are similar to the above subsections, and the program will be stopped when the condition $\quad\left(\left|\left\|f_{k+1}\right\|-\left\|f_{k}\right\|\right| /\left\|f_{k}\right\|\right)<10^{-3}$ or $\quad\left(\left|\left\|x_{k+1}\right\|-\left\|x_{k}\right\|\right| /\right.$ $\left.\left\|x_{k}\right\|\right)<10^{-3}$ holds. The following three images are selected as processing objects: Banoon $(512 \times 512)$, Barbara $(512 \times 512)$, and Lena $(512 \times 512)$. The detail performances are shown in Figures 7-9. The CPU time taken to process images is listed in Table 4.

It is easy to see that all the algorithms are successful for image restoration problems. The results in Table 4 reveal that the CPU time of Algorithm 1 is less than that of other algorithms, whether for $\$ 30 \backslash \% \$$ noise problems, $50 \%$ noise problems, or for $70 \%$ noise problems.

\section{Conclusion}

In this paper, a modified three-term type CD conjugate gradient algorithm is presented. Some good features are also presented: (i) sufficient descent property holds, (ii) trust region feature also holds, (iii) the algorithm has global convergence with the MWWP line search technique and projection technique for general function, and (iv) numerical results reveal that the new algorithm is more competitive than the normal CD algorithm and PRP algorithm. 
In recent years, there have been considerable researches about other types of CG method, while the study of CD method is not enough, and it should not be ignored. We have many works to do in the future: whether this method is suitable for other line search technique (such as Armijo line search, nonmonotone line search), or whether there exist other better modification methods to improve the numerical results of the CD method. All these are worth studying in the next work.

\section{Data Availability}

The data used to support the findings of this study are included within the article.

\section{Conflicts of Interest}

The authors declare that there are no conflicts of interest regarding the publication of this paper.

\section{Acknowledgments}

This work was supported by the National Natural Science Foundation of China (Grant no. 11661009), the High Level Innovation Teams and Excellent Scholars Program in Guangxi Institutions of Higher Education (Grant No.[2019] 52), and the Guangxi Natural Science Key Fund (No. 2017GXNSFDA198046). The authors would like to thank the editor and the referees for their valuable comments, which greatly improve this paper.

\section{References}

[1] M. Al-Baali, "Descent property and global convergence of the fletcher-reeves method with inexact line search," IMA Journal of Numerical Analysis, vol. 5, no. 1, pp. 121-124, 1985.

[2] Y. Dai and Y. Yuan, "A nonlinear conjugate gradient with a strong global convergence properties," SIAM Journal on Optimization, vol. 10, pp. 177-182, 2000.

[3] J. W. Daniel, "The conjugate gradient method for linear and nonlinear operator equations," SIAM Journal on Numerical Analysis, vol. 4, no. 1, pp. 10-26, 1967.

[4] R. Fletcher, Practical Methods of Optimization, Wiley, New York, NY, USA, 2nd. edition, 1987.

[5] R. Fletcher and C. M. Reeves, "Function minimization by conjugate gradients," The Computer Journal, vol. 7, no. 2, pp. 149-154, 1964.

[6] M. R. Hestenes and E. Stiefel, "Methods of conjugate gradients for solving linear systems," Journal of Research of the National Bureau of Standards, vol. 49, no. 6, pp. 409-436, 1952.

[7] Y. Liu and C. Storey, "Efficient generalized conjugate gradient algorithms, part 1: theory," Journal of Optimization Theory and Applications, vol. 69, no. 1, pp. 129-137, 1991.

[8] E. Polak and G. Ribiere, "Note sur la convergence de méthodes de directions conjuguées," Revue française d'informatique et de recherche opérationnelle. Série rouge, vol. 3, no. 16, pp. 35-43, 1969.

[9] B. T. Polyak, "The conjugate gradient method in extremal problems," USSR Computational Mathematics and Mathematical Physics, vol. 9, no. 4, pp. 94-112, 1969.

[10] G. Yuan, X. Wang, and Z. Sheng, "Family weak conjugate gradient algorithms and their convergence analysis for nonconvex functions," Numerical Algorithms, vol. 84, no. 3, pp. 935-956, 2020.

[11] S.-Y. Liu, Y.-Y. Huang, and H.-W. Jiao, "Sufficient descent conjugate gradient methods for solving convex constrained nonlinear monotone equations," Abstract and Applied Analysis, vol. 2014, pp. 1-12, 2014.

[12] X. Y. Wang, S. J. Li, and X. P. Kou, "A self-adaptive three-term conjugate gradient method for monotone nonlinear equations with convex constraints," Calcolo, vol. 53, no. 2, pp. 133-145, 2016.

[13] G. Yuan and W. Hu, "A conjugate gradient algorithm for large-scale unconstrained optimization problems and nonlinear equations," Journal of Inequalities and Applications, vol. 113, no. 1, 2018.

[14] G. Yuan, T. Li, and W. Hu, "A conjugate gradient algorithm for large-scale nonlinear equations and image restoration problems," Applied Numerical Mathematics, vol. 147, pp. 129-141, 2020.

[15] G. Yuan, J. Lu, and Z. Wang, "The PRP conjugate gradient algorithm with a modified WWP line search and its application in the image restoration problems," Applied Numerical Mathematics, vol. 152, pp. 1-11, 2020.

[16] G. Yuan, Z. Wei, and Y. Yang, "The global convergence of the polak-ribière-polyak conjugate gradient algorithm under inexact line search for nonconvex functions," Journal of Computational and Applied Mathematics, vol. 362, pp. 262275, 2019.

[17] L. Zhang, W. Zhou, and D.-H. Li, "A descent modified polakribière-polyak conjugate gradient method and its global convergence," IMA Journal of Numerical Analysis, vol. 26, no. 4, pp. 629-640, 2006.

[18] G. Yuan, W. Hu, and Z. Sheng, "A conjugate gradient algorithm with yuan-wei-lu line search," in International Conference on Cloud Computing and Security, Springer, Cham, Switzerland, 2017.

[19] G. Yuan, Z. Wei, and X. Lu, "Global convergence of BFGS and PRP methods under a modified weak Wolfe-Powell line search," Applied Mathematical Modelling, vol. 47, pp. 811825, 2017.

[20] G. Yuan, Z. Sheng, B. Wang, W. Hu, and C. Li, "The global convergence of a modified BFGS method for nonconvex functions," Journal of Computational and Applied Mathematics, vol. 327, pp. 274-294, 2018.

[21] Y. Yuan and W. Sun, Theory and Methods of Optimization, Science Press of China, Beijing, China, 1999.

[22] E. D. Dolan and J. J. Moré, "Benchmarking optimization software with performance profiles," Mathematical Programming, vol. 91, no. 2, pp. 201-213, 2002.

[23] A. Ouyang, L.-B. Liu, Z. Sheng, and F. Wu, "A class of parameter estimation methods for nonlinear muskingum model using hybrid invasive weed optimization algorithm," Mathematical Problems in Engineering, vol. 2015, pp. 1-15, 2015.

[24] A. Ouyang, Z. Tang, K. Li, A. Sallam, and E. Sha, "Estimating parameters of muskingum model using an adaptive hybrid PSO algorithm," International Journal of Pattern Recognition and Artificial Intelligence, vol. 28, pp. 1-29, 2014.

[25] Z. W. Geem, "Parameter estimation for the nonlinear muskingum model using the BFGS technique," Journal of Irrigation and Drainage Engineering, vol. 132, no. 5, pp. 474-478, 2006. 